\title{
Further Investigation of a Gaussian Sum Filter Based on Stochastic Equivalent Linearization*
}

\author{
Sueo Sugimoto ${ }^{\dagger}$, Yukihiro Kubo ${ }^{\dagger}$ and Masaharu OHASHI ${ }^{\dagger}$
}

\begin{abstract}
We proposed a stochastic (or statistical) Equivalent linearization - Gaussian Sum Filter(: EqGS Filter) for discrete time nonlinear Systems. Subsequently, in this paper, we investigate and show the further results related to the EqGS Filter. Especially we discuss a method to apply GaussHermite quadrature rules for evaluation of the conditional expected values of the quantities required to design the EqGS filter. Finally, we show the estimation results of AR modeling comparison with the extended Kalman and the equivalent linearization filters.
\end{abstract}

\section{Introduction}

Recently, many attentions again have been focused on nonlinear filtering theory $[1,2]$ and its approximated filters[3-5], such as the unscented Kalman filter[6], the particle (or Monte Carlo) filter[7,8], and the Gaussian filter[9,10]. We had proposed a stochastic (or statistical) Equivalent linearization - Gaussian Sum Filter (abbreviated as EqGS Filter) for discrete time nonlinear Systems in[11,12]. In this paper, we show the further results related to the EqGS Filter, especially we discuss a method of evaluation of the conditional expected values required to design the EqGS filter, by applying Gauss-Hermite quadrature rules[13-15]

Let us consider the following discrete-time nonlinear state space models.

$$
\begin{aligned}
x_{t+1} & =f_{t}\left(x_{t}\right)+w_{t} \\
y_{t} & =h_{t}\left(x_{t}\right)+v_{t}
\end{aligned}
$$

where $x_{t}$ is a $n$-dimensional state vector and $y_{t}$ is $m$-dimensional observation vector. $w_{t}$ and $v_{t}$ are $n$ dimensional and $m$-dimensional Gaussian white noises, respectively. $f_{t}\left(x_{t}\right)$ and $h_{t}\left(x_{t}\right)$ are $n$-dimensional and $m$-dimensional vector valued nonlinear functions. The mean vectors and covariance matrices of $w_{t}$ and $v_{t}$ are given by

$$
\begin{aligned}
& \mathrm{E}\left[w_{t}\right]=0, \quad \mathrm{E}\left[v_{t}\right]=0 \\
& \mathrm{E}\left\{\left[\begin{array}{l}
w_{t} \\
v_{t}
\end{array}\right]\left[\begin{array}{ll}
w_{s}^{\mathrm{T}} & \left.v_{s}^{\mathrm{T}}\right]
\end{array}\right]=\left[\begin{array}{cc}
Q_{t} & 0 \\
0 & R_{t}
\end{array}\right] \delta_{t-s}\right.
\end{aligned}
$$

\footnotetext{
* Manuscript Received Date: May 31, 2013
}

$\dagger$ Dept. of Electrical and Electronic Engineering, Ritsumeikan University; 1-1-1 Noji-Higashi, Kusatsu City, Shiga 525-8577, JAPAN

Key Words: nonlinear filter, equivalent linearization, Gaussian sum filter, Gauss-Hermite quadrature. and the initial state $x_{0}$ is independent of $w_{t}$ and $v_{t}$. Also due to whiteness of noises, we have the relations:

$$
\mathrm{E}\left\{w_{t} x_{s}^{\mathrm{T}}\right\}=0(t \geq s), \mathrm{E}\left\{v_{t} x_{s}^{\mathrm{T}}\right\}=0(t, s \geq 0) .
$$

\section{The Stochastic Equivalent Lineariza- tion}

The stochastic equivalent linearization technique[17] was applied to obtain the suboptimal linear estimates for continuous time systems by assuming the Gaussian conditional probability density functions (CPDFs). We had extended the equivalent linearization technique $[4,5,18]$ to discrete time systems under the assumption of Gaussian sum CPDFs: $p\left(x_{t} \mid Y^{t-1}\right), p\left(x_{t} \mid Y^{t}\right)$, where $Y^{t}=\left\{y_{0}, \ldots, y_{t}\right\}$. Now we show to derive the EqGS filter for discrete time nonlinear systems according to $[11,12]$.

First, we assume that the CPDFs are given by the following Gaussian sum distribution:

$$
\begin{aligned}
& p\left(x_{t} \mid Y^{t-1}\right) \\
= & \sum_{i=1}^{r} \alpha_{t \mid t-1}^{(i)}(2 \pi)^{-n / 2}\left|P_{t \mid t-1}^{(i)}\right|^{-1 / 2} \\
& \times \exp \left\{-\frac{1}{2}\left(x_{t}-\mu_{t \mid t-1}^{(i)}\right)^{\mathrm{T}}\left(P_{t \mid t-1}^{(i)}\right)^{-1}\left(x_{t}-\mu_{t \mid t-1}^{(i)}\right)\right\} \\
\equiv & \sum_{i=1}^{r} \alpha_{t \mid t-1}^{(i)} N\left(x_{t}: \mu_{t \mid t-1}^{(i)}, P_{t \mid t-1}^{(i)}\right), \\
& p\left(x_{t} \mid Y^{t}\right) \\
= & \sum_{i=1}^{r} \alpha_{t \mid t}^{(i)}(2 \pi)^{-n / 2}\left|P_{t \mid t}^{(i)}\right|^{-1 / 2} \\
& \times \exp \left\{-\frac{1}{2}\left(x_{t}-\mu_{t \mid t}^{(i)}\right)^{\mathrm{T}}\left(P_{t \mid t}^{(i)}\right)^{-1}\left(x_{t}-\mu_{t \mid t}^{(i)}\right)\right\} \\
\equiv & \sum_{i=1}^{r} \alpha_{t \mid t}^{(i)} N\left(x_{t}: \mu_{t \mid t}^{(i)}, P_{t \mid t}^{(i)}\right) .
\end{aligned}
$$

Namely, these CPDFs are Gaussian sum distribution 
with means: $\mu_{t \mid t-1}^{(i)}, \mu_{t \mid t}^{(i)} ; i=1, \cdots, r$ and covariances: $P_{t \mid t-1}^{(i)}, P_{t \mid t}^{(i)} ; i=1, \cdots, r$, where $\alpha_{t \mid t-1}^{(i)}$ and $\alpha_{t \mid t}^{(i)}$ denote weighting factors of distributions with the property, $\sum_{i=1}^{r} \alpha_{t \mid *}^{(i)}=1 ; \alpha_{t \mid *}^{(i)} \geq 0$.

Then we consider the linear approximation to the nonlinear functions: $f_{t}\left(x_{t}\right)$ and $h_{t}\left(x_{t}\right)$ in (1) and (2), respectively, as

$$
\begin{aligned}
& f_{t}\left(x_{t}\right)=a_{t}+\sum_{i=1}^{r} B_{t}^{(i)}\left(x_{t}-\mu_{t \mid t}^{(i)}\right)+e_{t}^{f}, \\
& h_{t}\left(x_{t}\right)=c_{t}+\sum_{i=1}^{r} D_{t}^{(i)}\left(x_{t}-\mu_{t \mid t-1}^{(i)}\right)+e_{t}^{h},
\end{aligned}
$$

where $a_{t}$ and $c_{t}$ are $n$-dimensional and $m$-dimensional vectors, respectively, and $B_{t}^{(i)}$ and $D_{t}^{(i)}$ are $n \times n$ and $m \times n$ matrices. Further, $e_{t}^{f}$ and $e_{t}^{h}$ denote the linear approximation errors. Similar to the stochastic equivalent linearization[17], we assume that the linear approximations are unbiased, namely

$$
\begin{aligned}
\mathrm{E}\left\{f_{t}\left(x_{t}\right) \mid Y^{t}\right\} & =\mathrm{E}\left\{a_{t}+\sum_{i=1}^{r} B_{t}^{(i)}\left(x_{t}-\mu_{t \mid t}^{(i)}\right) \mid Y^{t}\right\} \\
\mathrm{E}\left\{h_{t}\left(x_{t}\right) \mid Y^{t-1}\right\} & =\mathrm{E}\left\{c_{t}+\sum_{i=1}^{r} D_{t}^{(i)}\left(x_{t}-\mu_{t \mid t-1}^{(i)}\right) \mid Y^{t-1}\right\} .
\end{aligned}
$$

Furthermore, we consider the following optimization problem for minimizing the square error of linear approximation. Then $a_{t}, B_{t}^{(i)}, c_{t}$ and $D_{t}^{(i)}$ in (6) and (7) are obtained by minimizing the conditional expectation of the square errors norms of $e_{t}^{f}$ and $e_{t}^{h}$ :

$$
\begin{aligned}
& \mathrm{E}\left\{\left\|e_{t}^{f}\right\|^{2} \mid Y^{t}\right\} \equiv J_{f}\left(a_{t},\left\{B_{t}^{(i)}\right\}\right) \\
& \equiv \mathrm{E}\left\{\left\|f_{t}\left(x_{t}\right)-a_{t}-\sum_{i=1}^{r} B_{t}^{(i)}\left(x_{t}-\mu_{t \mid t}^{(i)}\right)\right\|^{2} \mid Y^{t}\right\} \\
& \mathrm{E}\left\{\left\|e_{t}^{h}\right\|^{2} \mid Y^{t-1}\right\} \equiv J_{h}\left(c_{t},\left\{D_{t}^{(i)}\right\}\right) \\
& \quad \equiv \mathrm{E}\left\{\left\|h_{t}\left(x_{t}\right)-c_{t}-\sum_{i=1}^{r} D_{t}^{(i)}\left(x_{t}-\mu_{t \mid t-1}^{(i)}\right)\right\|^{2} \mid Y^{t-1}\right\}
\end{aligned}
$$

Then we compute (10) as follows:

$$
\begin{aligned}
& J_{f}\left(a_{t},\left\{B_{t}^{(i)}\right\}\right) \equiv J_{f}\left(a_{t}, B_{t}\right) \\
& =\operatorname{trace}\left[\mathrm { E } \left\{\left[f_{t}\left(x_{t}\right)-a_{t}-\sum_{i=1}^{r} B_{t}^{(i)}\left(x_{t}-\mu_{t \mid t}^{(i)}\right)\right]\right.\right. \\
& \left.\left.\quad \times\left[f_{t}\left(x_{t}\right)-a_{t}-\sum_{j=1}^{r} B_{t}^{(j)}\left(x_{t}-\mu_{t \mid t}^{(j)}\right)\right]^{\mathrm{T}} \mid Y^{t}\right\}\right] \\
& =\operatorname{trace}\left[\mathrm { E } \left\{\left(f_{t}\left(x_{t}\right)-a_{t}\right)\left(f_{t}\left(x_{t}\right)-a_{t}\right)^{\mathrm{T}}\right.\right. \\
& \quad+\sum_{i=1}^{r} \sum_{j=1}^{r} B_{t}^{(i)}\left(x_{t}-\mu_{t \mid t}^{(i)}\right)\left(x_{t}-\mu_{t \mid t}^{(j)}\right)^{\mathrm{T}}\left(B_{t}^{(j)}\right)^{\mathrm{T}} \\
& \quad-\sum_{j=1}^{r}\left(f_{t}\left(x_{t}\right)-a_{t}\right)\left(x_{t}-\mu_{t \mid t}^{(j)}\right)^{\mathrm{T}}\left(B_{t}^{(j)}\right)^{\mathrm{T}}
\end{aligned}
$$

$$
\begin{aligned}
& \left.\left.-\sum_{i=1}^{r} B_{t}^{(i)}\left(x_{t}-\mu_{t \mid t}^{(i)}\right)\left(f_{t}\left(x_{t}\right)-a_{t}\right)^{\mathrm{T}} \mid Y^{t}\right\}\right] \\
= & \operatorname{trace}\left[a_{t} a_{t}^{\mathrm{T}}-\mathrm{E}\left\{f_{t}\left(x_{t}\right) \mid Y^{t}\right\} a_{t}^{\mathrm{T}}-a_{t} \mathrm{E}\left\{f_{t}^{\mathrm{T}}\left(x_{t}\right) \mid Y^{t}\right\}\right. \\
& +\mathrm{E}\left\{f_{t}\left(x_{t}\right) f_{t}^{\mathrm{T}}\left(x_{t}\right) \mid Y^{t}\right\}+\sum_{i=1}^{r} \sum_{j=1}^{r} B_{t}^{(i)} F_{t}^{(i j)}\left(B_{t}^{(j)}\right)^{\mathrm{T}} \\
& \left.-\sum_{j=1}^{r} \Gamma_{t}^{(j)}\left(B_{t}^{(j)}\right)^{\mathrm{T}}-\sum_{i=1}^{r} B_{t}^{(i)}\left(\Gamma_{t}^{(i)}\right)^{\mathrm{T}}\right] \\
= & \operatorname{trace}\left[\left(a_{t}-\mathrm{E}\left\{f_{t}\left(x_{t}\right) \mid Y^{t}\right\}\right)\left(a_{t}-\mathrm{E}\left\{f_{t}\left(x_{t}\right) \mid Y^{t}\right\}\right)^{\mathrm{T}}\right. \\
& +\mathrm{E}\left\{f_{t}\left(x_{t}\right) f_{t}^{\mathrm{T}}\left(x_{t}\right) \mid Y^{t}\right\}-\mathrm{E}\left\{f_{t}\left(x_{t}\right) \mid Y^{t}\right\} \mathrm{E}\left\{f_{t}^{\mathrm{T}}\left(x_{t}\right) \mid Y^{t}\right\} \\
& \left.+B_{t} F_{t} B_{t}^{\mathrm{T}}-\Gamma_{t} B_{t}^{\mathrm{T}}-B_{t} \Gamma_{t}^{\mathrm{T}}\right]
\end{aligned}
$$

where

$$
\begin{aligned}
& B_{t} \equiv\left[\begin{array}{llll}
B_{t}^{(1)} & B_{t}^{(2)} & \cdots & B_{t}^{(r)}
\end{array}\right] ; n \times n r \text { matrix } \\
& F_{t} \equiv\left[\begin{array}{ccc}
F_{t}^{(11)} & \cdots & F_{t}^{(1 r)} \\
\vdots & \vdots & \vdots \\
F_{t}^{(r 1)} & \cdots & F_{t}^{(r r)}
\end{array}\right] ; n r \times n r \text { matrix } \\
& F_{t}^{(i j)} \equiv \mathrm{E}\left\{\left(x_{t}-\mu_{t \mid t}^{(i)}\right)\left(x_{t}-\mu_{t \mid t}^{(j)}\right)^{\mathrm{T}} \mid Y^{t}\right\} \\
& \Gamma_{t} \equiv\left[\begin{array}{llll}
\Gamma_{t}^{(1)} & \Gamma_{t}^{(2)} & \cdots & \Gamma_{t}^{(r)}
\end{array}\right] ; n \times n r \text { matrix } \\
& \Gamma_{t}^{(i)} \equiv \mathrm{E}\left\{\left(f_{t}\left(x_{t}\right)-a_{t}\right)\left(x_{t}-\mu_{t \mid t}^{(i)}\right)^{\mathrm{T}} \mid Y^{t}\right\} \text {. }
\end{aligned}
$$

From (14) and (15), we have the relation:

$$
F_{t}^{\mathrm{T}}=F_{t}, \quad \text { since } \quad\left(F_{t}^{(i j)}\right)^{\mathrm{T}}=F_{t}^{(j i)} .
$$

Then minimization of $J_{f}\left(a_{t}, B_{t}\right)$ in (12) can be achieved by solving the following equations:

$$
\begin{aligned}
& \frac{\partial J_{f}\left(a_{t}, B_{t}\right)}{\partial a_{t, i}}=0, \\
& \frac{\partial J_{f}\left(a_{t}, B_{t}\right)}{\partial B_{t, i j}}=0,
\end{aligned}
$$

for $i=1, \cdots, n ; j=1, \cdots, n r$. Then from (19), we have

$$
a_{t}=\mathrm{E}\left\{f_{t}\left(x_{t}\right) \mid Y^{t}\right\} \equiv \hat{f}_{t \mid t}\left(x_{t}\right),
$$

Also remarking the relations:

$$
\begin{aligned}
\operatorname{trace}\left[B_{t} F_{t} B_{t}^{\mathrm{T}}\right] & =\sum_{k} \sum_{p} \sum_{l} B_{t, k l} F_{t, l p} B_{t, k p}, \\
\operatorname{trace}\left[\Gamma_{t} B_{t}^{\mathrm{T}}\right] & =\sum_{k} \sum_{l} \Gamma_{t, k l} B_{t, k l}, \\
\operatorname{trace}\left[B_{t} \Gamma_{t}^{\mathrm{T}}\right] & =\sum_{k} \sum_{l} B_{t, k l} \Gamma_{t, k l},
\end{aligned}
$$

then from (20), we have

$$
2 \sum_{p} F_{t, j p} B_{t, i p}-2 \Gamma_{t, i j}=0 .
$$

Namely, we have

$$
B_{t} F_{t}=\Gamma_{t} .
$$


Also from (8), we have

$$
\begin{aligned}
\mathrm{E}\left\{f_{t}\left(x_{t}\right) \mid Y^{t}\right\} & =a_{t}+\sum_{i=1}^{r} B_{t}^{(i)}\left[\mathrm{E}\left\{x_{t} \mid Y^{t}\right\}-\mu_{t \mid t}^{(i)}\right] \\
& =a_{t}+B_{t}\left(\hat{X}_{t \mid t}-\mu_{t \mid t}\right)
\end{aligned}
$$

where

$$
\begin{aligned}
\hat{X}_{t \mid t} & \equiv\left[\begin{array}{lll}
\hat{x}_{t \mid t}^{\mathrm{T}} & \cdots & \hat{x}_{t \mid t}^{\mathrm{T}}
\end{array}\right]^{\mathrm{T}} ; n r \times 1 \text { vector } \\
\mu_{t \mid t} & \equiv\left[\begin{array}{lll}
\left(\mu_{t \mid t}^{(1)}\right)^{\mathrm{T}} & \cdots & \left(\mu_{t \mid t}^{(r)}\right)^{\mathrm{T}}
\end{array}\right]^{\mathrm{T}} ; n r \times 1 \text { vector. }
\end{aligned}
$$

From $(21)$ and $(24)$, we have $B_{t}\left(\hat{X}_{t \mid t}-\mu_{t \mid t}\right)=0$, so that combining with (23), we have

$$
B_{t}\left[F_{t} \mid \hat{X}_{t \mid t}-\mu_{t \mid t}\right]=\left[\Gamma_{t} \mid 0_{n \times 1}\right]
$$

Then from (27), we finally have

$$
B_{t}=\tilde{\Gamma}_{t} \tilde{F}_{t}^{\mathrm{T}}\left(\tilde{F}_{t} \tilde{F}_{t}^{\mathrm{T}}\right)^{-1}
$$

where

$$
\tilde{F}_{t} \equiv\left[F_{t} \mid \hat{X}_{t \mid t}-\mu_{t \mid t}\right], \quad \tilde{\Gamma}_{t} \equiv\left[\Gamma_{t} \mid 0_{n \times 1}\right] .
$$

Also from (17) and (21), we should remark the relations:

$$
\begin{aligned}
\Gamma_{t}^{(i)} & =\mathrm{E}\left\{\left(f_{t}\left(x_{t}\right)-a_{t}\right) x_{t}^{\mathrm{T}} \mid Y^{t}\right\} \\
& =\mathrm{E}\left\{f_{t}\left(x_{t}\right) x_{t}^{\mathrm{T}} \mid Y^{t}\right\}-a_{t} \hat{x}_{t \mid t}^{\mathrm{T}} \equiv \Gamma_{t}^{(0)} .
\end{aligned}
$$

Therefore we can approximate

$$
\begin{aligned}
f_{t}\left(x_{t}\right) & \approx a_{t}+\sum_{i=1}^{r} B_{t}^{(i)}\left(x_{t}-\mu_{t \mid t}^{(i)}\right) \\
& =a_{S, t}+B_{S, t} x_{t}
\end{aligned}
$$

where

$$
\begin{aligned}
a_{S, t} & \equiv a_{t}-\sum_{i=1}^{r} B_{t}^{(i)} \mu_{t \mid t}^{(i)}=a_{t}-B_{t} \mu_{t \mid t} \\
B_{S, t} & \equiv \sum_{i=1}^{r} B_{t}^{(i)} .
\end{aligned}
$$

Similarly, $c_{t}, D_{t}$ are obtained by

$$
\begin{aligned}
& c_{t}=\mathrm{E}\left\{h_{t}\left(x_{t}\right) \mid Y^{t-1}\right\} \equiv \hat{h}_{t \mid t-1}\left(x_{t}\right) \\
& D_{t}\left[H_{t} \mid\left(\hat{X}_{t \mid t-1}-\mu_{t \mid t-1}\right)\right]=\left[\Phi_{t} \mid 0_{m \times 1}\right]
\end{aligned}
$$

where

$$
\begin{aligned}
& D_{t}=\left[\begin{array}{llll}
D_{t}^{(1)} & D_{t}^{(2)} & \cdots & D_{t}^{(r)}
\end{array}\right] ; m \times n r \text { matrix } \\
& H_{t}=\left[\begin{array}{ccc}
H_{t}^{(11)} & \cdots & H_{t}^{(1 r)} \\
\vdots & \vdots & \vdots \\
H_{t}^{(r 1)} & \cdots & H_{t}^{(r r)}
\end{array}\right] ; n r \times n r \text { matrix } \\
& H_{t}^{(i j)} \equiv \mathrm{E}\left\{\left(x_{t}-\mu_{t \mid t-1}^{(i)}\right)\left(x_{t}-\mu_{t \mid t-1}^{(j)}\right)^{\mathrm{T}} \mid Y^{t-1}\right\} \\
& \Phi_{t}=\left[\begin{array}{llll}
\Phi_{0, t} & \Phi_{0, t} & \cdots & \Phi_{0, t}
\end{array}\right] ; m \times n r \text { matrix }
\end{aligned}
$$

$$
\Phi_{0, t} \equiv \mathrm{E}\left\{h_{t}\left(x_{t}\right) x_{t}^{\mathrm{T}} \mid Y^{t-1}\right\}-c_{t} \hat{x}_{t \mid t-1}^{\mathrm{T}} .
$$

Then, similar to (31), we can approximate

$$
\begin{aligned}
h_{t}\left(x_{t}\right) \approx c_{t}+\sum_{i=1}^{r} D_{t}^{(i)}\left(x_{t}-\mu_{t \mid t-1}^{(i)}\right) \\
\equiv c_{S, t}+D_{S, t} x_{t} \\
c_{S, t} \equiv c_{t}-\sum_{i=1}^{r} D_{t}^{(i)} \mu_{t \mid t-1}^{(i)} \\
D_{S, t} \equiv \sum_{i=1}^{r} D_{t}^{(i)}: \quad m \times n .
\end{aligned}
$$

Therefore from (31) and (41), the nonlinear state space models in (1), (2) are approximated by the quasilinear equations:

$$
\begin{aligned}
x_{t+1} & =B_{S, t} x_{t}+a_{S, t}+w_{t} \\
y_{t} & =D_{S, t} x_{t}+c_{S, t}+v_{t} .
\end{aligned}
$$

\section{EqGS Filter}

We now present a new and promising nonlinear filter by combining the Gaussian sum filter and the stochastic linearization technique. Similar to the derivation of the Gaussian sum filter, we apply the Bayesian rule to the $\operatorname{CPDF} p\left(x_{t} \mid Y^{t}\right)$ as follows[19]:

$$
\begin{aligned}
& p\left(x_{t} \mid Y^{t}\right) \\
& =\frac{p\left(x_{t}, Y^{t}\right)}{p\left(Y^{t}\right)}=\frac{p\left(x_{t}, Y^{t-1}, y_{t}\right)}{p\left(Y^{t-1}, y_{t}\right)} \\
& =\frac{p\left(y_{t} \mid x_{t}\right) p\left(x_{t} \mid Y^{t-1}\right)}{p\left(y_{t} \mid Y^{t-1}\right)}=\frac{p\left(x_{t} \mid Y^{t-1}\right) p\left(y_{t} \mid x_{t}\right)}{\int p\left(x_{t} \mid Y^{t-1}\right) p\left(y_{t} \mid x_{t}\right) d x_{t}} \\
& =\frac{\sum_{i=1}^{r} \alpha_{t \mid t-1}^{(i)} N\left(x_{t}: \mu_{t \mid t-1}^{(i)}, P_{t \mid t-1}^{(i)}\right) p\left(y_{t}-h_{t}\left(x_{t}\right)\right)}{\int \sum_{i=1}^{r} \alpha_{t \mid t-1}^{(i)} N\left(x_{t}: \mu_{t \mid t-1}^{(i)}, P_{t \mid t-1}^{(i)}\right) p\left(y_{t}-h_{t}\left(x_{t}\right)\right) d x_{t}} \\
& \approx \frac{\sum_{i=1}^{r} \alpha_{t \mid t-1}^{(i)} N\left(x_{t}: \mu_{t \mid t-1}^{(i)}, P_{t \mid t-1}^{(i)}\right) N\left(\xi_{t}: 0, R_{t}\right)}{\int \sum_{i=1}^{r} \alpha_{t \mid t-1}^{(i)} N\left(x_{t}: \mu_{t \mid t-1}^{(i)}, P_{t \mid t-1}^{(i)}\right) N\left(\xi_{t}: 0, R_{t}\right) d x_{t}}
\end{aligned}
$$

where

$$
\begin{aligned}
y_{t}-h_{t}\left(x_{t}\right) \approx & \xi_{t} \\
\equiv & y_{t}-D_{S, t} x_{t}-c_{S, t}=d_{t}-D_{S, t} x_{t}, \\
& d_{t} \equiv y_{t}-c_{S, t} .
\end{aligned}
$$

For evaluating (46), we use the relation:

$$
\begin{aligned}
& N\left(x_{t}: \mu_{t \mid t-1}^{(i)}, P_{t \mid t-1}^{(i)}\right) N\left(\xi_{t}: 0, R_{t}\right) \\
& =\frac{e^{-\frac{1}{2}\left[\left(x_{t}-\mu_{t \mid t-1}^{(i)}\right)^{\mathrm{T}}\left(P_{t \mid t-1}^{(i)}\right)^{-1}\left(x_{t}-\mu_{t \mid t-1}^{(i)}\right)+\xi_{t}^{\mathrm{T}} R_{t}^{-1} \xi_{t}\right]}}{(\sqrt{2 \pi})^{n+m}\left|P_{t \mid t-1}^{(i)}\right|^{1 / 2}\left|R_{t}\right|^{1 / 2}} .
\end{aligned}
$$

Then the power term of the above exponential function is completed by the square as follows. (Here and hereafter the lower subscripts are sometimes omitted, due to the limited space.)

$$
\left(x_{t}-\mu_{t \mid t-1}^{(i)}\right)^{\mathrm{T}}\left(P_{t \mid t-1}^{(i)}\right)^{-1}\left(x_{t}-\mu_{t \mid t-1}^{(i)}\right)+\xi_{t}^{\mathrm{T}} R_{t}^{-1} \xi_{t}
$$




$$
\begin{aligned}
\equiv & \left(x-\mu^{(i)}\right)^{\mathrm{T}}\left(P^{(i)}\right)^{-1}\left(x-\mu^{(i)}\right) \\
& +\left(d-D_{S} x\right)^{\mathrm{T}} R^{-1}\left(d-D_{S} x\right) \\
= & {\left[x-\left(\left(P^{(i)}\right)^{-1}+D_{S}^{\mathrm{T}} R^{-1} D_{S}\right)^{-1}\right.} \\
& \left.\times\left(\left(P^{(i)}\right)^{-1} \mu^{(i)}+D_{S}^{\mathrm{T}} R^{-1} d\right)\right]^{\mathrm{T}} \\
& \times\left(\left(P^{(i)}\right)^{-1}+D_{S}^{\mathrm{T}} R^{-1} D_{S}\right) \\
& \times\left[x-\left(\left(P^{(i)}\right)^{-1}+D_{S}^{\mathrm{T}} R^{-1} D_{S}\right)^{-1}\right. \\
& \left.\times\left(\left(P^{(i)}\right)^{-1} \mu^{(i)}+D_{S}^{\mathrm{T}} R^{-1} d\right)\right] \\
& -\left(\left(P^{(i)}\right)^{-1} \mu^{(i)}+D_{S}^{\mathrm{T}} R^{-1} d\right)^{\mathrm{T}} \\
& \times\left(\left(P^{(i)}\right)^{-1}+D_{S}^{\mathrm{T}} R^{-1} D_{S}\right) \\
& \times\left(\left(P^{(i)}\right)^{-1} \mu^{(i)}+D_{S}^{\mathrm{T}} R^{-1} d\right) \\
& +\left(\mu^{(i)}\right)^{\mathrm{T}}\left(P^{(i)}\right)^{-1} \mu^{(i)}+d^{\mathrm{T}} R^{-1} d .
\end{aligned}
$$

Further, for the terms after the second term at the right-hand side in (50), we proceed to complete the square with respect to $d$, which contains the observation $y$ in (48). Namely, define

$$
W^{(i)} \equiv\left[\left(P^{(i)}\right)^{-1}+D_{S}^{\mathrm{T}} R^{-1} D_{S}\right]^{-1},
$$

then, the terms after the second term in (50) are described by (52) which appears at the top of the next page, Therefore, from (49), (50) and (52), we have the relation:

$$
\begin{aligned}
& N\left(x_{t}: \mu_{t \mid t-1}^{(i)}, P_{t \mid t-1}^{(i)}\right) N\left(\xi_{t}: 0, R_{t}\right) \\
& \quad=k_{t}^{(i)} N\left(x_{t}: m_{x_{t}}^{(i)}, \Sigma_{x_{t}}^{(i)}\right) N\left(y_{t}: m_{y_{t}}^{(i)}, \Sigma_{y_{t}}^{(i)}\right)
\end{aligned}
$$

where

$$
\begin{aligned}
k_{t}^{(i)} & \equiv \frac{\left|\left[\left(P^{(i)}\right)^{-1}+D_{S}^{\mathrm{T}} R_{t}^{-1} D_{S}\right]^{-1}\right|^{1 / 2}\left|R_{t}+D_{S} P^{(i)} D_{S}^{\mathrm{T}}\right|^{1 / 2}}{\left|P_{t \mid t-1}^{(i)}\right|^{1 / 2}\left|R_{t}\right|^{1 / 2}} \\
m_{x_{t}}^{(i)} & \equiv\left[D_{S, t}^{\mathrm{T}} R_{t}^{-1} D_{S, t}+\left(P_{t \mid t-1}^{(i)}\right)^{-1}\right]^{-1} \\
\quad \times\left[\left(P_{t \mid t-1}^{(i)}\right)^{-1} \mu_{t \mid t-1}^{(i)}+D_{S, t}^{\mathrm{T}} R_{t}^{-1} d_{t}\right] & \\
\Sigma_{x t}^{(i)} & \equiv\left[\left(P_{t \mid t-1}^{(i)}\right)^{-1}+D_{S, t}^{\mathrm{T}} R_{t}^{-1} D_{S, t}\right]^{-1} \\
m_{y t}^{(i)} & \equiv c_{S, t}+D_{S, t} \mu_{t \mid t-1}^{(i)} \\
\Sigma_{y t}^{(i)} & \equiv D_{S, t} P_{t \mid t-1}^{(i)} D_{S, t}^{\mathrm{T}}+R_{t} .
\end{aligned}
$$

Here we assume the Gaussian sum form for (46):

$$
p\left(x_{t} \mid Y^{t}\right)=\sum_{i=1}^{r} \alpha_{t \mid t}^{(i)} N\left(x_{t}: \mu_{t \mid t}^{(i)}, P_{t \mid t}^{(i)}\right),
$$

then, from (46), (53)

$$
\begin{aligned}
& \sum_{i=1}^{r} \alpha_{t \mid t}^{(i)} N\left(x_{t}: \mu_{t \mid t}^{(i)}, P_{t \mid t}^{(i)}\right) \\
\approx & \frac{\sum_{i=1}^{r} \alpha_{t \mid t-1}^{(i)} k_{t}^{(i)} N\left(x_{t}: m_{x_{t}}^{(i)}, \Sigma_{x_{t}}^{(i)}\right) N\left(y_{t}: m_{y_{t}}^{(i)}, \Sigma_{y_{t}}^{(i)}\right)}{\int \sum_{j=1}^{r} \alpha_{t \mid t-1}^{(j)} k_{t}^{(j)} N\left(x_{t}: m_{x_{t}}^{(j)}, \Sigma_{x_{t}}^{(j)}\right) N\left(y_{t}: m_{y_{t}}^{(j)}, \Sigma_{y_{t}}^{(j)}\right) d x_{t}} \\
= & \frac{\sum_{i=1}^{r} \alpha_{t \mid t-1}^{(i)} k_{t}^{(i)} N\left(x_{t}: m_{x_{t}}^{(i)}, \Sigma_{x_{t}}^{(i)}\right) N\left(y_{t}: m_{y_{t}}^{(i)}, \Sigma_{y_{t}}^{(i)}\right)}{\sum_{j=1}^{r} \alpha_{t \mid t-1}^{(j)} k_{t}^{(j)} N\left(y_{t}: m_{y_{t}}^{(j)}, \Sigma_{y_{t}}^{(j)}\right)}
\end{aligned}
$$

Then by applying the matrix inversion lemma, we have

$$
\begin{aligned}
\mu_{t \mid t}^{(i)}= & m_{x_{t}}^{(i)} \\
= & {\left[\left(P_{t \mid t-1}^{(i)}\right)^{-1}+D_{S}^{\mathrm{T}} R^{-1} D_{S}\right]^{-1} } \\
& \times\left[\left(P_{t \mid t-1}^{(i)}\right)^{-1} \mu_{t \mid t-1}^{(i)}+D_{S}^{\mathrm{T}} R^{-1}\left(y-c_{S}\right)\right] \\
= & \mu_{t \mid t-1}^{(i)}+\left[\left(P_{t \mid t-1}^{(i)}\right)^{-1}+D_{S}^{\mathrm{T}} R^{-1} D_{S}\right]^{-1} \\
& \quad \times D_{S}^{\mathrm{T}} R^{-1}\left(y-c_{S}-D_{S} \mu_{t \mid t-1}^{(i)}\right) \\
= & \mu_{t \mid t-1}^{(i)}+\left[P_{t \mid t-1}^{(i)}-P_{t \mid t-1}^{(i)} D_{S}^{\mathrm{T}}\left(D_{S} P_{t \mid t-1}^{(i)} D_{S}^{\mathrm{T}}\right.\right. \\
& \left.+R)^{-1} D_{S} P_{t \mid t-1}^{(i)}\right] D_{S}^{\mathrm{T}} R^{-1}\left(y-c_{S}-D_{S} \mu_{t \mid t-1}^{(i)}\right) \\
= & \mu_{t \mid t-1}^{(i)}+\left[P_{t \mid t-1}^{(i)}-K_{t}^{(i)} D_{S, t} P_{t \mid t-1}^{(i)}\right] D_{S, t}^{\mathrm{T}} R^{-1} \\
& \quad \times\left(y_{t}-c_{S, t}-D_{S, t} \mu_{t \mid t-1}^{(i)}\right) .
\end{aligned}
$$

Also we have the relation

$$
\begin{aligned}
P_{t \mid t}^{(i)}= & \Sigma_{x_{t}}^{(i)} \\
= & {\left[\left(P_{t \mid t-1}^{(i)}\right)^{-1}+D_{S, t}^{\mathrm{T}} R_{t}^{-1} D_{S, t}\right]^{-1} } \\
= & P_{t \mid t-1}^{(i)}-P_{t \mid t-1}^{(i)} D_{S, t}^{\mathrm{T}} \\
& \quad \times\left(D_{S, t} P_{t \mid t-1}^{(i)} D_{S, t}^{\mathrm{T}}+R_{t}\right)^{-1} D_{S, t} P_{t \mid t-1}^{(i)} .
\end{aligned}
$$

Then finally we have the following equations:

$$
\begin{aligned}
& \mu_{t \mid t}^{(i)}=\mu_{t \mid t-1}^{(i)}+\left[P_{t \mid t-1}^{(i)}-K_{t}^{(i)} D_{S, t} P_{t \mid t-1}^{(i)}\right] \times D_{S, t}^{\mathrm{T}} R_{t}^{-1}\left(y_{t}-c_{S, t}-D_{S, t} \mu_{t \mid t-1}^{(i)}\right) \\
& P_{t \mid t}^{(i)}=P_{t \mid t-1}^{(i)}-K_{t}^{(i)} D_{S, t} P_{t \mid t-1}^{(i)} \\
& K_{t}^{(i)} \equiv P_{t \mid t-1}^{(i)} D_{S, t}^{\mathrm{T}}\left[D_{S, t} P_{t \mid t-1}^{(i)} D_{S, t}^{\mathrm{T}}+R_{t}\right]^{-1}
\end{aligned}
$$

Also from (55), we have

$$
\begin{aligned}
\alpha_{t \mid t}^{(i)} & \approx \frac{\alpha_{t \mid t-1}^{(i)} k_{t}^{(i)} N\left(y_{t}: m_{y_{t}}^{(i)}, \Sigma_{y_{t}}^{(i)}\right)}{\sum_{j=1}^{r} \alpha_{t \mid t-1}^{(j)} k_{t}^{(j)} N\left(y_{t}: m_{y_{t}}^{(j)}, \Sigma_{y_{t}}^{(j)}\right)} \\
& \equiv \frac{\alpha_{t \mid t-1}^{(i)} \beta_{t}^{(i)}}{\sum_{j=1}^{r} \alpha_{t \mid t-1}^{(j)} \beta_{t}^{(j)}},
\end{aligned}
$$

where

$$
\begin{aligned}
\beta_{t}^{(i)} \equiv & k_{t}^{(i)} N\left(y_{t}: c_{S, t}+D_{S, t} \mu_{t \mid t-1}^{(i)}, D_{S, t} P_{t \mid t-1}^{(i)} D_{S, t}^{\mathrm{T}}+R\right) \\
= & \frac{\left|\left[\left(P_{t \mid t-1}^{(i)}\right)^{-1}+D_{S}^{\mathrm{T}} R_{t}^{-1} D_{S}\right]^{-1}\right|^{1 / 2}}{(\sqrt{2 \pi})^{m}\left|P_{t \mid t-1}^{(i)}\right|^{1 / 2}\left|R_{t}\right|^{1 / 2}} \\
& \times e^{-\frac{1}{2}\left[\left(y-c_{S}-D_{S} \mu\right)^{\mathrm{T}}\left(D_{S} P D_{S}^{\mathrm{T}}+R\right)^{-1}\left(y-c_{S}-D_{S} \mu\right)\right]}
\end{aligned}
$$

Therefore the filtered estimate can be computed by

$$
\begin{aligned}
\hat{x}_{t \mid t} & =\mathrm{E}\left[x_{t} \mid Y^{t}\right]=\int_{-\infty}^{\infty} x_{t} p\left(x_{t} \mid Y^{t}\right) d x_{t} \\
& =\int_{-\infty}^{\infty} x_{t} \sum_{i=1}^{r} \alpha_{t \mid t}^{(i)} N\left(x_{t}: \mu_{t \mid t}^{(i)}, P_{i, t \mid t}\right) d x_{t} \\
& =\sum_{i=1}^{r} \alpha_{t \mid t}^{(i)} \mu_{t \mid t}^{(i)} .
\end{aligned}
$$




$$
\begin{aligned}
& -\left[\left(P^{(i)}\right)^{-1} \mu^{(i)}+D_{S}^{\mathrm{T}} R^{-1} d\right]^{\mathrm{T}}\left[\left(P^{(i)}\right)^{-1}+D_{S}^{\mathrm{T}} R^{-1} D_{S}\right]^{-1}\left[\left(P^{(i)}\right)^{-1} \mu^{(i)}+D_{S}^{\mathrm{T}} R^{-1} d\right]+\left(\mu^{(i)}\right)^{\mathrm{T}}\left(P^{(i)}\right)^{-1} \mu^{(i)}+d^{\mathrm{T}} R^{-1} d \\
= & -\left[\left(\mu^{(i)}\right)^{\mathrm{T}}\left(P^{(i)}\right)^{-1}+d^{\mathrm{T}} R^{-1} D_{S}\right] W^{(i)}\left[\left(P^{(i)}\right)^{-1} \mu^{(i)}+D_{S}^{\mathrm{T}} R^{-1} d\right]+\left(\mu^{(i)}\right)^{\mathrm{T}}\left(P^{(i)}\right)^{-1} \mu^{(i)}+d^{\mathrm{T}} R^{-1} d \\
= & {\left[d-\left(R^{-1}-R^{-1} D_{S} W^{(i)} D_{S}^{\mathrm{T}} R^{-1}\right)^{-1} R^{-1} D_{S} W^{(i)}\left(P^{(i)}\right)^{-1} \mu^{(i)}\right]^{\mathrm{T}}\left(R^{-1}-R^{-1} D_{S} W^{(i)} D_{S}^{\mathrm{T}} R^{-1}\right) } \\
& \times\left[d-\left(R^{-1}-R^{-1} D_{S} W^{(i)} D_{S}^{\mathrm{T}} R^{-1}\right)^{-1} R^{-1} D_{S} W^{(i)}\left(P^{(i)}\right)^{-1} \mu^{(i)}\right] \\
& +\left(\mu^{(i)}\right)^{\mathrm{T}}\left[\left(P^{(i)}\right)^{-1}-\left(P^{(i)}\right)^{-1} W^{(i)}\left(P^{(i)}\right)^{-1}-\left(P^{(i)}\right)^{-1} W^{(i)} D_{S}^{\mathrm{T}} R^{-1}\left(R^{-1}-R^{-1} D_{S} W^{(i)} D_{S}^{\mathrm{T}} R^{-1}\right)^{-1} R^{-1} D_{S} W^{(i)}\left(P^{(i)}\right)^{-1}\right] \mu^{(i)} \\
= & \left(d-D_{S} \mu^{(i)}\right)^{\mathrm{T}}\left(R+D_{S} P^{(i)} D_{S}^{\mathrm{T}}\right)^{-1}\left(d-D_{S} \mu^{(i)}\right)=\left(y-c_{S}-D_{S} \mu^{(i)}\right)^{\mathrm{T}}\left(R+D_{S} P^{(i)} D_{S}^{\mathrm{T}}\right)^{-1}\left(y-c_{S}-D_{S} \mu^{(i)}\right),
\end{aligned}
$$

where we have applied the matrix inversion lemma: $R^{-1}-R^{-1} D_{S} W^{(i)} D_{S}^{\mathrm{T}} R^{-1}=\left(R+D_{S} P^{(i)} D_{S}^{\mathrm{T}}\right)^{-1}$, and the relations:

$$
\begin{aligned}
& \left(R^{-1}-R^{-1} D_{S} W^{(i)} D_{S}^{\mathrm{T}} R^{-1}\right)^{-1} R^{-1} D_{S} W^{(i)}\left(P^{(i)}\right)^{-1}=\left(R+D_{S} P^{(i)} D_{S}^{\mathrm{T}}\right) R^{-1} D_{S}\left[\left(P^{(i)}\right)^{-1}+D_{S}^{\mathrm{T}} R^{-1} D_{S}\right]^{-1}\left(P^{(i)}\right)^{-1} \\
& =\left(D_{S}+D_{S} P^{(i)} D_{S}^{\mathrm{T}} R^{-1} D_{S}\right)\left(I+P^{(i)} D_{S}^{\mathrm{T}} R^{-1} D_{S}\right)^{-1}=D_{S} \quad(\text { appear on 3rd and 4th lines in }(52)), \\
\text { and } & \\
& \left(P^{(i)}\right)^{-1}-\left(P^{(i)}\right)^{-1} W^{(i)}\left(P^{(i)}\right)^{-1}-\left(P^{(i)}\right)^{-1} W^{(i)} D_{S}^{\mathrm{T}} R^{-1} D_{S} \\
& =\left(P^{(i)}\right)^{-1}-\left(P^{(i)}\right)^{-1}\left(\left(P^{(i)}\right)^{-1}+D_{S}^{\mathrm{T}} R^{-1} D_{S}\right)^{-1}\left(P^{(i)}\right)^{-1}-\left(P^{(i)}\right)^{-1}\left(\left(P^{(i)}\right)^{-1}+D_{S}^{\mathrm{T}} R^{-1} D_{S}\right)^{-1} D_{S}^{\mathrm{T}} R^{-1} D_{S} \\
& \left.=\left(P^{(i)}\right)^{-1}-\left(P^{(i)}\right)^{-1}\left[\left(P^{(i)}\right)^{-1}+D_{S}^{\mathrm{T}} R^{-1} D_{S}\right]^{-1}\left(\left(P^{(i)}\right)^{-1}+D_{S}^{\mathrm{T}} R^{-1} D_{S}\right)=O \quad \text { (appear on the 5th line in }(52)\right) .
\end{aligned}
$$

Also the filtered error covariance matrix $P_{t \mid t}$ is given by

$$
\begin{aligned}
P_{t \mid t} & \equiv \mathrm{E}\left\{\left(x_{t}-\hat{x}_{t \mid t}\right)\left(x_{t}-\hat{x}_{t \mid t}\right)^{\mathrm{T}} \mid Y^{t}\right\} \\
& =\mathrm{E}\left\{x_{t} x_{t}^{\mathrm{T}} \mid Y^{t}\right\}-\hat{x}_{t \mid t} \hat{x}_{t \mid t}^{\mathrm{T}} \\
& =\sum_{i=1}^{r} \alpha_{t \mid t}^{(i)}\left(P_{t \mid t}^{(i)}+\mu_{t \mid t}^{(i)}\left(\mu_{t \mid t}^{(i)}\right)^{\mathrm{T}}\right)-\hat{x}_{t \mid t} \hat{x}_{t \mid t}^{\mathrm{T}} .
\end{aligned}
$$

Therefore the quasi-linear filter with Gaussian sum CPDFs is summarized as the following formula of updating:

$$
\hat{x}_{t \mid t}=\sum_{i=1}^{r} \alpha_{t \mid t}^{(i)} \mu_{t \mid t}^{(i)},
$$

with (57)-(61).

\section{Time updating formula}

Let us derive the time updating formula as follows. Define

$$
\hat{x}_{t+1 \mid t} \equiv \sum_{i=1}^{r} \alpha_{t+1 \mid t}^{(i)} \mu_{t+1 \mid t}^{(i)} .
$$

Also from (44), we have the relations:

$$
\begin{aligned}
\hat{x}_{t+1 \mid t} & =B_{S, t} \hat{x}_{t \mid t}+a_{S, t} \\
& =B_{S, t} \sum_{i=1}^{r} \alpha_{t \mid t}^{(i)} \mu_{t+1 \mid t}^{(i)}+a_{S, t} \\
& =\sum_{i=1}^{r} \alpha_{t \mid t}^{(i)}\left[B_{S, t} \mu_{t \mid t}^{(i)}+a_{S, t}\right],
\end{aligned}
$$

where we use the relation: $\sum_{i=1}^{r} \alpha_{t \mid t}^{(i)}=1$. Comparing (66) with (65), we have

$$
\alpha_{t+1 \mid t}^{(i)}=\alpha_{t \mid t}^{(i)}
$$

$$
\mu_{t+1 \mid t}^{(i)}=a_{S, t}+B_{S, t} \mu_{t \mid t}^{(i)} .
$$

Also define

$$
P_{t+1 \mid t}^{(i)} \equiv \mathrm{E}\left\{\left[x_{t+1}-\mu_{t+1 \mid t}^{(i)}\right]\left[x_{t+1}-\mu_{t+1 \mid t}^{(i)}\right] \mid Y^{t}\right\}^{\mathrm{T}}
$$

then from (44) and (68), we have

$$
x_{t+1}-\mu_{t+1 \mid t}^{(i)}=B_{S, t} x_{t}+w_{t}-B_{S, t} \mu_{t \mid t}^{(i)} .
$$

Therefore, from (69) and (70), we have the relation:

$$
P_{t+1 \mid t}^{(i)}=B_{S, t} P_{t \mid t}^{(i)} B_{S, t}^{\mathrm{T}}+Q_{t}
$$

Then, however, we need to obtain $a_{t},\left\{B_{t}^{(i)}\right\}, c_{t}$, $\left\{D_{t}^{(i)}\right\}$ such that we should evaluate the values of

$$
\begin{aligned}
\mathrm{E}\left[f_{t}\left(x_{t}\right) \mid Y^{t}\right], & \mathrm{E}\left[f_{t}\left(x_{t}\right) x_{t}^{\mathrm{T}} \mid Y^{t}\right] \\
\mathrm{E}\left[h_{t}\left(x_{t}\right) \mid Y^{t-1}\right], & \mathrm{E}\left[h_{t}\left(x_{t}\right) x_{t}^{\mathrm{T}} \mid Y^{t-1}\right]
\end{aligned}
$$

which appear in (21), (27), (34) and (35).

(Remark 1) In the case of the system noise $w_{t}$ having the Gaussian sum distribution[16], namely,

$$
p\left(w_{t}\right) \equiv \sum_{i=1}^{q_{t}} \gamma_{t}^{(i)} N\left(w_{t}: \eta_{t}^{(i)}, Q_{t}^{(i)}\right)
$$

we had derived the similar form of the EqGS filter (see[12]).

\section{Evaluation of Conditional Expected Values}

Our EqGS filter requires to evaluate the expectation of (72) under the assumption of the conditional Gaussian (or, Gaussian sum) PDF. For this purpose, we have already shown a method to evaluate the higher moments by applying the moment generating function[12]. Here we briefly show the method to 
evaluate the moments of the vector random variable which have Gaussain distributions.

Namely, let the CPDF for $n$-dimensional Gaussian random vector $x=\left[\begin{array}{llll}x_{1} & x_{2} & \cdots & x_{n}\end{array}\right]^{\mathrm{T}}$ is described by

$$
\begin{aligned}
p(x \mid Y)= & \frac{1}{(2 \pi)^{n / 2}|\Sigma|^{1 / 2}} \\
& \times \exp \left\{-\frac{1}{2}(x-\mu)^{\mathrm{T}} \Sigma^{-1}(x-\mu)\right\},
\end{aligned}
$$

where $\mu$ and $\Sigma$ are conditional mean and conditional covariance matrix, respectively, as follows

$$
\begin{aligned}
\mu & =\mathrm{E}[x \mid Y] \\
\Sigma & =\mathrm{E}\left[(x-\mu)(x-\mu)^{\mathrm{T}} \mid Y\right] \\
& =\left[\begin{array}{rrr}
\sigma_{11} & \cdots & \sigma_{1 n} \\
\vdots & \ddots & \vdots \\
\sigma_{n 1} & \cdots & \sigma_{n n}
\end{array}\right] .
\end{aligned}
$$

Let $s=\left[\begin{array}{llll}s_{1} & s_{2} & \cdots & s_{n}\end{array}\right]^{\mathrm{T}}$, then it is well known that the moment generating function for the multivariate normal distribution is given by

$$
M(s) \equiv \mathrm{E}\left[e^{s^{\mathrm{T}} x} \mid Y\right]=\exp \left\{s^{\mathrm{T}} \mu+\frac{1}{2} s^{\mathrm{T}} \Sigma s\right\}
$$

For example, we can evaluate the conditional expectation of a fifth moment $\mathrm{E}\left[x_{\nu_{1}} x_{\nu_{2}} x_{\nu_{3}} x_{\nu_{4}} x_{\nu_{5}} \mid Y\right]$ as follows. Namely, applying the relation,

$$
\left.\frac{\partial^{5} M(s)}{\partial s_{\nu_{1}} \partial s_{\nu_{2}} \partial s_{\nu_{3}} \partial s_{\nu_{4}} \partial s_{\nu_{5}}}\right|_{s=0}=\mathrm{E}\left[x_{\nu_{1}} x_{\nu_{2}} x_{\nu_{3}} x_{\nu_{4}} x_{\nu_{5}} \mid Y\right]
$$

we have

$$
\begin{aligned}
E & {\left[x_{\nu_{1}} x_{\nu_{2}} \cdots x_{\nu_{5}} \mid Y^{t}\right] } \\
& =\sigma_{\nu_{1} \nu_{2}} \sigma_{\nu_{3} \nu_{4}} \mu_{\nu_{5}}+\sigma_{\nu_{1} \nu_{2}} \sigma_{\nu_{3} \nu_{5}} \mu_{\nu_{4}}+\sigma_{\nu_{1} \nu_{2}} \sigma_{\nu_{4} \nu_{5}} \mu_{\nu_{3}} \\
& +\sigma_{\nu_{1} \nu_{3}} \sigma_{\nu_{2} \nu_{4}} \mu_{\nu_{5}}+\sigma_{\nu_{1} \nu_{3}} \sigma_{\nu_{2} \nu_{5}} \mu_{\nu_{4}}+\sigma_{\nu_{1} \nu_{3}} \sigma_{\nu_{4} \nu_{5}} \mu_{\nu_{2}} \\
& +\sigma_{\nu_{1} \nu_{4}} \sigma_{\nu_{2} \nu_{3}} \mu_{\nu_{5}}+\sigma_{\nu_{1} \nu_{4}} \sigma_{\nu_{2} \nu_{5}} \mu_{\nu_{3}}+\sigma_{\nu_{1} \nu_{4}} \sigma_{\nu_{3} \nu_{5}} \mu_{\nu_{2}} \\
& +\sigma_{\nu_{1} \nu_{5}} \sigma_{\nu_{2} \nu_{3}} \mu_{\nu_{4}}+\sigma_{\nu_{1} \nu_{5}} \sigma_{\nu_{2} \nu_{4}} \mu_{\nu_{3}}+\sigma_{\nu_{1} \nu_{5}} \sigma_{\nu_{3} \nu_{4}} \mu_{\nu_{2}} \\
& +\sigma_{\nu_{2} \nu_{3}} \sigma_{\nu_{4} \nu_{5}} \mu_{\nu_{1}}+\sigma_{\nu_{2} \nu_{4}} \sigma_{\nu_{3} \nu_{5}} \mu_{\nu_{1}}+\sigma_{\nu_{2} \nu_{5}} \sigma_{\nu_{3} \nu_{4}} \mu_{\nu_{1}} \\
& +\sigma_{\nu_{1} \nu_{2}} \mu_{\nu_{3}} \mu_{\nu_{4}} \mu_{\nu_{5}}+\sigma_{\nu_{1} \nu_{3}} \mu_{\nu_{2}} \mu_{\nu_{4}} \mu_{\nu_{5}}+\sigma_{\nu_{1} \nu_{4}} \mu_{\nu_{2}} \mu_{\nu_{3}} \mu_{\nu_{5}} \\
& +\sigma_{\nu_{1} \nu_{5}} \mu_{\nu_{2}} \mu_{\nu_{3}} \mu_{\nu_{4}}+\sigma_{\nu_{2} \nu_{3}} \mu_{\nu_{1}} \mu_{\nu_{4}} \mu_{\nu_{5}}+\sigma_{\nu_{2} \nu_{4}} \mu_{\nu_{1}} \mu_{\nu_{3}} \mu_{\nu_{5}} \\
& +\sigma_{\nu_{2} \nu_{5}} \mu_{\nu_{1}} \mu_{\nu_{3}} \mu_{\nu_{4}}+\sigma_{\nu_{3} \nu_{4}} \mu_{\nu_{1}} \mu_{\nu_{2}} \mu_{\nu_{5}}+\sigma_{\nu_{3} \nu_{5}} \mu_{\nu_{1}} \mu_{\nu_{2}} \mu_{\nu_{4}} \\
& +\sigma_{\nu_{4} \nu_{5}} \mu_{\nu_{1}} \mu_{\nu_{2}} \mu_{\nu_{3}}+\mu_{\nu_{1}} \mu_{\nu_{2}} \mu_{\nu_{3}} \mu_{\nu_{4}} \mu_{\nu_{5}} .
\end{aligned}
$$

The numerical integration technique consist of approximating the integrand by a polynomial in a region or regions. Namely, a complicated integral of $h(x)$ is factored into a nonnegative weight function $\omega(x)$ and another function $g(x)$ :

$$
\int_{a}^{b} h(x) d x=\int_{a}^{b} \omega(x) g(x) d x \approx \sum_{j=1}^{N} w_{j} g\left(z_{j}\right) .
$$

Then we have the following integration rules called as Gaussian quadrature rules.

\section{Gaussian Quadrature Rules[13]}

Let $\omega(x) \geq 0$ be a weight function defined on $[a, b]$. For $\omega(x)$, it is possible to define a sequence of orthonormal polynomials $q_{1}(x), q_{2}(x), \cdots$ with respect to $\omega(x)$ and in which $q_{j}(x)$ is of exact degree $j$ so that

$$
\int_{a}^{b} q_{j}(x) q_{k}(x) \omega(x) d x=\left\{\begin{array}{l}
1 ; j=k \\
0 ; j \neq k
\end{array}\right.
$$

The polynominal

$$
q_{m}(x)=k_{m} \prod_{j=1}^{m}\left(x-z_{j}\right), k_{m}>0
$$

has $m$ real roots $a<z_{1}<z_{2}<\cdots<z_{m}<b$. Then we have the following theorems[13,14].

\section{[Theorem 1]}

Let $g(x) \in C^{2 m}[a, b]$, then

$$
\begin{gathered}
\int_{a}^{b} \omega(x) g(x) d x=\sum_{j=1}^{m} w_{j} g\left(z_{j}\right)+\frac{g^{(2 m)}(\xi)}{(2 m) ! k_{m}^{2}} \\
(a<\xi<b)
\end{gathered}
$$

where

$$
w_{j}=-\frac{k_{m+1}}{k_{m}} \frac{1}{q_{m+1}\left(z_{j}\right) q_{m}^{\prime}\left(z_{j}\right)}
$$

Thus the Gauss quadrature rule is exact for all polynomials of degree $\leq 2 m-1$.

\section{[Theorem 2]}

Let us consider any set of orthonormal polynomials $\left\{q_{j}(x)\right\}_{j=1}^{m}$, satisfying a three recurrence relationship:

$$
\begin{aligned}
q_{j}(x)= & \left(a_{j} x+b_{j}\right) q_{j-1}(x)-c_{j} q_{j-2}(x), \\
& j=1, \cdots, m \\
& q_{-1}(x) \equiv 0, \quad q_{0}(x) \equiv 1,
\end{aligned}
$$

with $a_{j}>0$ and $c_{j}>0$. Then (80) is described by the matrix equation:

$$
x \mathbf{q}(x)=T \mathbf{q}(x)+\frac{1}{a_{m}} q_{m}(x) \mathbf{e}_{m}
$$

where $T$ is the tridiagonal matrix;

$$
T \equiv\left[\begin{array}{ccccccc}
-\frac{b_{1}}{a_{1}} & \frac{1}{a_{1}} & 0 & \ldots & \ldots & \ldots & 0 \\
\frac{c_{2}}{a_{2}} & -\frac{b_{2}}{a_{2}} & \frac{1}{a_{2}} & 0 & \ldots & \ldots & 0 \\
0 & \ddots & \ddots & \ddots & & \vdots \\
\vdots & & & & \ddots & 0 \\
\vdots & & & \ddots & \ddots & \frac{1}{a_{m}} \\
0 & \cdots & \cdots & 0 & \frac{c_{m}}{a_{m}} & -\frac{b_{m}}{a_{m}}
\end{array}\right]
$$

and

$$
\begin{aligned}
\mathbf{q}(x) & \equiv\left[q_{0}(x), \cdots, q_{m-1}(x)\right]^{\mathrm{T}} \\
\mathbf{e}_{m} & \equiv[0, \cdots, 0,1]^{\mathrm{T}} .
\end{aligned}
$$

Then $\left\{z_{j} ; j=1,2, \cdots, m\right\}$ are eigenvalues of $T$ and $\left\{w_{j} ; j=1,2, \cdots, m\right\}$ equal to $\left|\left(q_{j}\right)_{1}\right|^{2}$ where $\left(q_{j}\right)_{1}$ is the first element of $j$ th normalized eigenvector of $T$. 
Namely,

$$
\begin{aligned}
z_{j} \mathbf{q}\left(z_{j}\right) & =T \mathbf{q}\left(z_{j}\right) \\
\mathbf{q}^{\mathrm{T}}\left(z_{j}\right) \mathbf{q}\left(z_{j}\right) & =1
\end{aligned}
$$

Let us now consider the Gaussian-type numerical integration of a scalor function $g(x)$. Namely,

$$
\int_{-\infty}^{\infty} g(x) \omega(x) d x=\sum_{i=1}^{m} w_{i} g\left(z_{i}\right)
$$

where

$$
\omega(x)=e^{-x^{2}}
$$

The conditional expectation of the other nonlinear functions of $x_{t}$ in (72) as well as the polynomials can be numerically computed by applying Gauss-Hermite quadrature rules $[13,9,10,15]$.

\section{Gauss-Hermite Quadrature Scheme}

It is well known that Hermite polynomials $\left\{H_{j}(x)\right.$; $j=1, \cdots, m\}$ are given by the following three term recurrence relationship:

$$
\begin{aligned}
H_{j}(x)= & 2 x H_{j-1}-(j-1) H_{j-2}, \\
& H_{-1}(x) \equiv 0, \quad H_{0}(x) \equiv 1,
\end{aligned}
$$

and

$$
\int_{-\infty}^{\infty} H_{j}(x) H_{k}(x) e^{-x^{2}} d x=\delta_{j-k} 2^{j} j ! \sqrt{\pi} .
$$

Thus the normalized weight $\omega(x)$ is given by

$$
\omega(x)=\frac{1}{\sqrt{\pi}} e^{-x^{2}}, \quad \text { due to } \quad \int_{-\infty}^{\infty} \omega(x) d x=1
$$

From (92), the corresponding normalized Hermite polynomial $\left\{\tilde{H}_{j}(x) ; j=1, \cdots, m\right\}$ are also given by

$$
\tilde{H}_{j}(x)=\frac{1}{\sqrt{2^{j} j !}} H_{j}(x),
$$

or

$$
H_{j}(x)=\sqrt{2^{j} j !} \tilde{H}_{j}(x) .
$$

Substituting (95) into (90), we have the three term recurrence equations regarding to $\left\{\tilde{H}_{j}(x) ; j=1, \cdots, m\right\}$ as follows:

$$
\begin{aligned}
& \tilde{H}_{j}(x)= \sqrt{\frac{2}{j}} x \tilde{H}_{j-1}-\sqrt{\frac{j-1}{j}} \tilde{H}_{j-2}, \\
& \tilde{H}_{-1}(x) \equiv 0, \quad \tilde{H}_{0}(x) \equiv 1
\end{aligned}
$$

Thus, in (80)

$$
a_{j}=\sqrt{\frac{2}{j}}, \quad b_{j}=0, \quad c_{j}=\sqrt{\frac{j-1}{j}}
$$

such that in $(83)$

$$
\frac{c_{j+1}}{a_{j+1}}=\sqrt{\frac{j}{2}}=\frac{1}{a_{j}}, \quad i=1, \cdots, m-1 .
$$

Therefore, $T$ in (83) becomes the symmetric tridiagonal matrix with zero diagonals and $T_{j, j+1}=\sqrt{j / 2}, j=$ $1, \cdots, m-1$. Then the expected value of $F(t)$ with $n$ dimensional Gaussian PDF $N(t: \mu, \Sigma)[9]$ :

$$
\begin{aligned}
I= & \int_{R^{n}} F(t) \frac{1}{\left((2 \pi)^{n} \operatorname{det} \Sigma\right)^{1 / 2}} \\
& \times \exp \left\{-\frac{1}{2}(t-\mu)^{\mathrm{T}} \Sigma^{-1}(t-\mu)\right\} d t
\end{aligned}
$$

can be evaluated as follows.

Let $\Sigma=S^{\mathrm{T}} S$ and $t=\mu+\sqrt{2} S^{\mathrm{T}} z$, then

$$
\begin{aligned}
& -\frac{1}{2}(t-\mu)^{\mathrm{T}} \Sigma^{-1}(t-\mu) \\
= & -\frac{1}{2} \sqrt{2} z^{\mathrm{T}} S S^{-1} S^{-\mathrm{T}} \sqrt{2} S^{-\mathrm{T}} z=-z^{\mathrm{T}} z=-\|z\|^{2}
\end{aligned}
$$

and $d t=(\sqrt{2})^{n} \operatorname{det} S d z$. Therefore, we have

$$
\begin{aligned}
I= & \int_{R^{n}} F\left(\mu+\sqrt{2} S^{\mathrm{T}} z\right) \\
& \times \frac{1}{(\sqrt{2 \pi})^{n} \operatorname{det} S} e^{-\|z\|^{2}(\sqrt{2})^{n} \operatorname{det} S d z} \\
= & \int_{R^{n}}^{m} F\left(\mu+\sqrt{2} S^{\mathrm{T}} z\right) \frac{1}{(\sqrt{\pi})^{n}} e^{-\|z\|^{2}} d z \\
\approx & \sum_{j_{1}=1}^{m} \cdots \sum_{j_{n}=1}^{m} F\left(\mu+\sqrt{2} S^{\mathrm{T}}\left[z_{j_{1}}, \ldots, z_{j_{n}}\right]^{\mathrm{T}}\right) w_{j_{1}} \cdots w_{j_{n}}
\end{aligned}
$$

By calculating the eigenvalues and the corresponding normalized eigenvectors of $T$ in (83) with (98) and (99), we have and $z_{1}=2 / \sqrt{2}, z_{1}=-2 / \sqrt{2}$ and $w_{1}=$ $w_{2}=1 / 2$ for $m=2 ; z_{1}=0, z_{2}=\sqrt{3 / 2}, z_{2}=-\sqrt{3 / 2}$ and $w_{1}=2 / 3, w_{2}=w_{3}=1 / 6$ for $m=3$.

\section{Numerical Examples of AR(1) Modeling}

Let us show $\mathrm{AR}(1)$ modeling by applying EqGS filter, and show what kinds of evaluation for conditional expected values are required.

Consider a $\mathrm{AR}(1)$ model with a linear measurement equation as follows:

$$
\begin{aligned}
x_{t+1}= & a x_{t}+w_{t} \\
y_{t}= & x_{t}+v_{t} \\
& w \sim N(0, Q), \quad v \sim N(0, R) .
\end{aligned}
$$

Then let $x_{1, t} \equiv x_{t}, x_{2, t} \equiv a$, we have the state and measurement equations:

$$
\begin{aligned}
{\left[\begin{array}{c}
x_{1, t+1} \\
x_{2, t+1}
\end{array}\right] } & =\left[\begin{array}{c}
x_{1, t} x_{2, t} \\
x_{2, t}
\end{array}\right]+\left[\begin{array}{l}
1 \\
0
\end{array}\right] w_{t} \\
& =f\left(x_{t}\right)+e_{t} w_{t}
\end{aligned}
$$

and

$$
y_{t}=\left[\begin{array}{ll}
1 & 0
\end{array}\right]\left[\begin{array}{l}
x_{1, t} \\
x_{2, t}
\end{array}\right]+v_{t}
$$


From (21), we have

$$
\begin{aligned}
a_{t} & =\mathrm{E}\left\{f\left(x_{t}\right) \mid Y^{t}\right\} \\
& =\sum_{i=1}^{r} \alpha_{t \mid t}^{(i)}\left[\begin{array}{c}
\mu_{1, t \mid t}^{(i)} \mu_{2, t \mid t}^{(i)}++P_{12, t \mid t}^{(i)} \\
\mu_{1, t \mid t}^{(i)}
\end{array}\right]
\end{aligned}
$$

Then also from (15)

$$
\begin{aligned}
F_{t}^{(i j)}= & P_{t \mid t}+\hat{x}_{t \mid t} \hat{x}_{t \mid t}^{\mathrm{T}}-\hat{x}_{t \mid t}\left(\mu_{t \mid t}^{(j)}\right)^{\mathrm{T}} \\
& -\mu_{t \mid t}^{(i)} \hat{x}_{t \mid t}^{\mathrm{T}}+\mu_{t \mid t}^{(i)}\left(\mu_{t \mid t}^{(j)}\right)^{\mathrm{T}} .
\end{aligned}
$$

From (17), we have

$$
\Gamma_{t}^{(0)}=\mathrm{E}\left\{f\left(x_{t}\right) x_{t}^{\mathrm{T}} \mid Y^{t}\right\}-a_{t} \sum_{k=1}^{r} \alpha_{t \mid t}^{(k)}\left(\mu_{t \mid t}^{(k)}\right)^{\mathrm{T}}
$$

The first term of the right hand side in (107) is given by

$$
\begin{aligned}
& \mathrm{E}\left\{f\left(x_{t}\right) x_{t}^{\mathrm{T}} \mid Y^{t}\right\} \\
& \quad=\mathrm{E}\left\{\left[\begin{array}{c}
x_{1, t} x_{2, t} \\
x_{2, t}
\end{array}\right]\left[x_{1, t} x_{2, t}\right] \mid Y^{t}\right\}
\end{aligned}
$$

These conditional expected values can be evaluated, for example, the $(1,1)$ element:

$$
\begin{aligned}
\mathrm{E} & \left\{x_{1, t}^{2} x_{2, t} \mid Y^{t}\right\} \\
& =\sum_{k=1}^{r} \alpha_{t \mid t}^{(k)}\left[2 \mu_{1}^{(k)} P_{12}^{(k)}+\mu_{2}^{(k)} P_{11}^{(k)}+\left(\mu_{1}^{(k)}\right)^{\mathrm{T}} \mu_{2}^{(k)}\right]
\end{aligned}
$$

On the other hand, the measurement equation is linear in this model, therefore in (57)-(61) we have

$$
D_{s, t}=H_{t} \equiv\left[\begin{array}{ll}
1 & 0
\end{array}\right] \text {. }
$$

Then from (62)

$$
\hat{x}_{t \mid t}=\mathrm{E}\left\{x_{t} \mid Y^{t}\right\}=\sum_{k=1}^{r} \alpha_{t \mid t}^{(k)} \mu_{t \mid t}^{(k)}
$$

We show simulation results of EqGS filter by comparing with the extended Kalman filter (EKF) and the equivalent linearization filter $(\mathrm{EqF})$. As is mentioned in Section 2, EqGS filter is derived by extending $\mathrm{EqF}$ with the Gaussian sum assumptions, therefore the algorithm of $\mathrm{EqF}$ can be given by assuming that the CDPDFs in (6) are Gaussian, i.e. $r=1$.

For simulations, we assumed that $a=0.96, Q=1$ and $x_{1,0}=0$. In this case, $\operatorname{Var}\left(x_{1, t}\right)=1 /\left(1-0.96^{2}\right)=$ 12.75 , and we assumed $R=12.75\left(\equiv R_{0}\right)$. For EqGS filter, the number of Gaussian sum was 3, i.e. $r=3$, and the initial values were assumed to be

$$
\begin{aligned}
& \hat{x}_{0 \mid-1}^{(1)}=\left[\begin{array}{r}
0 \\
-1
\end{array}\right], \hat{x}_{0 \mid-1}^{(2)}=\left[\begin{array}{l}
0 \\
0
\end{array}\right], \hat{x}_{0 \mid-1}^{(3)}=\left[\begin{array}{l}
0 \\
1
\end{array}\right], \\
& P_{0 \mid-1}^{(1)}=P_{0 \mid-1}^{(2)}=P_{0 \mid-1}^{(3)}=\operatorname{diag}\left(12.75,0.5^{2}\right), \\
& \alpha_{0 \mid-1}^{(1)}=\alpha_{0 \mid-1}^{(2)}=\alpha_{0 \mid-1}^{(3)}=1 / 3 .
\end{aligned}
$$

From (110)-(112), the initial values for EKF and EqF were given as follows.

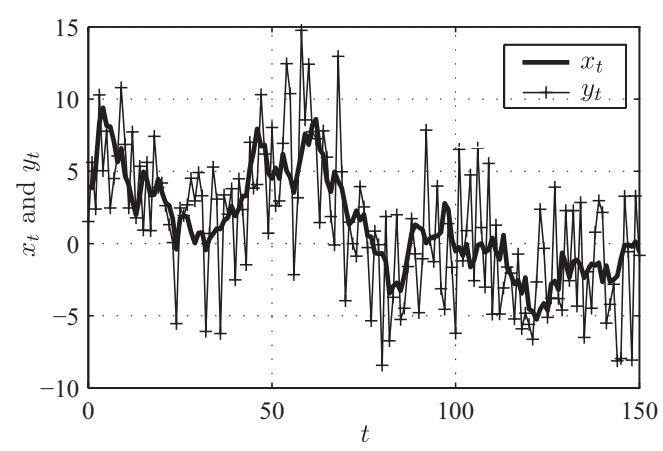

Fig. 1 A sample of true state and observation processes

$$
\begin{aligned}
\hat{x}_{0 \mid-1} & =\sum_{k=1}^{3} \alpha_{0 \mid-1}^{(k)} \hat{x}_{0 \mid-1}^{(k)}=\left[\begin{array}{l}
0 \\
0
\end{array}\right], \\
P_{0 \mid-1} & =\operatorname{diag}(12.75,0.917) .
\end{aligned}
$$

Fig. 1 shows the sample trajectories of the true state and observation processes. And Fig. 2 (a), (b) and (c) show the estimation results by EKF, EqF and EqGSF, respectively. To compare the performance of filters, 50 simulations with different trajectories were carried out, and the root mean square errors defined by the following formula were evaluated.

$$
E_{i, t}=\sqrt{\frac{1}{50} \sum_{j=1}^{50}\left(x_{i, t}^{\{j\}}-\hat{x}_{i, t \mid t}^{\{j\}}\right)^{2}}, \quad i=1,2,
$$

where $\{j\}$ indicates the $j$-th run in 50 simulations. Table 1 shows the parameter estimation performance of $\hat{x}_{2,150 \mid 150}$. From Table 1 it can be seen that the performance of EqF and EqGSF are superior to EKF.

To evaluate the filter performance in noisy environment, similar simulations were carried out for $R=$ $0.1 R_{0}, 0.5 R_{0}, 1.5 R_{0}, 2 R_{0}$, and $E_{2,150}$ values are plotted in Fig. 3. From Fig. 3 we can see that EqF and EqGSF show superior estimation results than EKF when the signal to noise ratio is lower ( $R$ is larger).

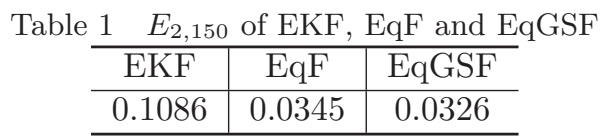

\section{Conclusions}

We have shown the Gaussian Sum filter based on the stochastic Equivalent linearization (: EqGS filter) for discrete time nonlinear systems. Furthermore we discuss two methods to evaluate the conditional expected values of quantities having Gaussian distribution which are required to derive Eq and EqGS filters. Also throughout the numerical experiments, we show that from the aspect of accuracy of estimation, the EqGS filter is superior among three filters when measurement noise become larger. However we should point out that our EqGS filter requires a little heavy computational cost compare to others. 

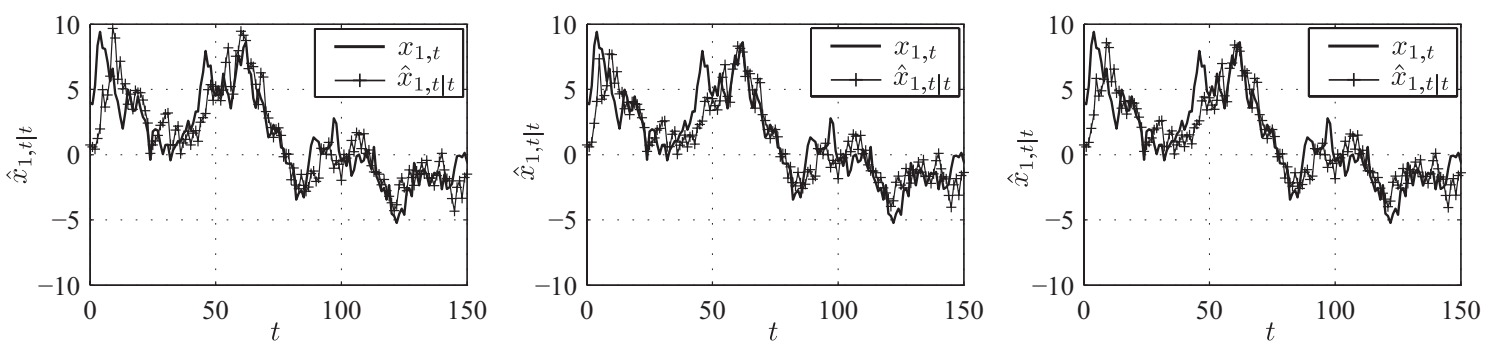

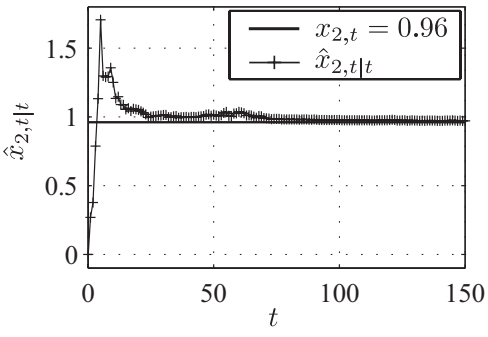

(a) EKF

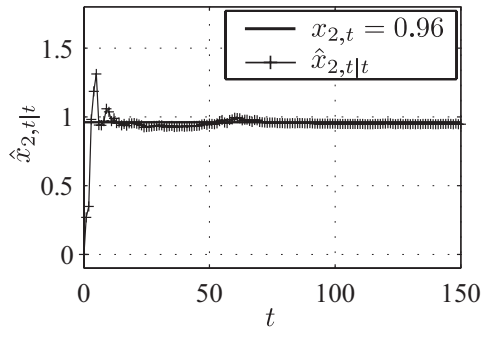

(b) $\mathrm{EqF}$

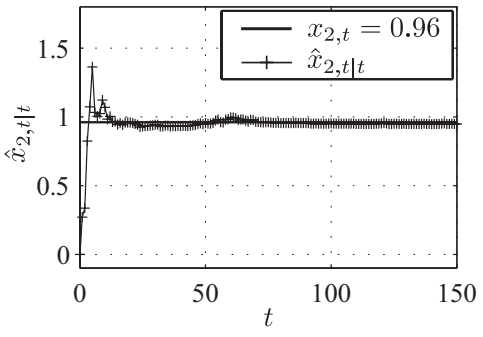

(c) EqGSF

Fig. 2 Estimation results by EKF, EqF and EqGSF

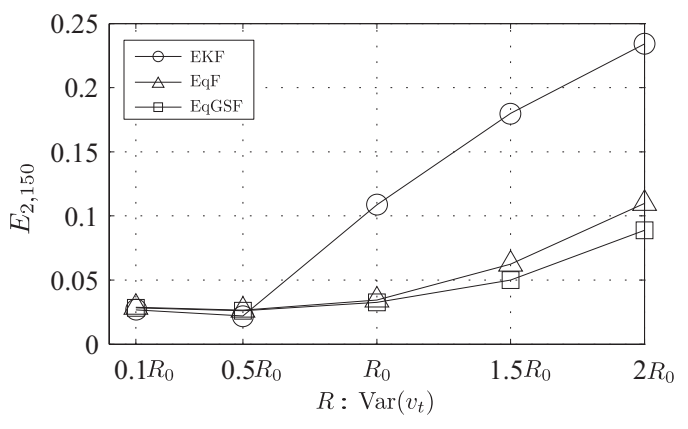

Fig. $3 E_{2,150}$ with respect to variations in $R$

\section{References}

[1]H. J. Kushner: Approximation to optimal filters; IEEE Trans. on Automatic Control, Vol. AC-12, No. 5, pp. 546-556 (1967)

[2]A. H. Jazwinski: Stochastic Process and Filtering Theory, Academic (1970)

[3]S. Sugimoto, Y. Kubo and M. Tanikawara: A review and applications of the nonlinear filters to GNSS/INS integrated algorithms; Proc. the Institute of Navigation, ION GNSS 2009, pp. 3101-3113 (2009)

[4]S. Sugimoto and M. Tanigawara: Probability - statistics and Kalman filter, Appendix 4; GPS Handbook, edited by S. Sugimoto and R. Shibasaki, pp. 432-460 Asakura-Shoten (2010) (in Japanese)

[5]T. Katayama: Nonlinear Kalman Filter, AsakuraShoten (2011) (in Japanese)

[6]S. J. Julier and J. K. Uhlmann: Unscented filtering and nonlinear estimation; Proceedings of the IEEE, Vol. 92, No. 3 (2004)

[7]G. Kitagawa: Monte Carlo filter and smoother for non-Gaussian nonlinear state space models; Jounal of Computational and Graphical Statistics, Vol. 5, pp. 125 (1996)

[8]W. Gersch and G. Kitagawa: General state space modeling; Statistical Methods in Control and Signal Processing, T. Katayama and S. Sugimoto, eds., Marcel Dekker, pp. 37-81 (1997)
[9]K. Ito and K. Xiong: Gaussian filters for nonlinear filtering problems; IEEE Trans. on Automatic Control, Vol. 45, No. 5, pp. 910-927 (2000)

[10]I. Arasaratnam and S. Haykin: Discrete-time nonlinear filtering algorithms using Gauss-Hermite quadrature; Proc. IEEE, Vol. 95, No. 5, pp. 953-977 (2007)

[11]S. Sugimoto and Y. Kubo: A Gaussian sum filter based on stochastic equivalent linearization; Proc. the 41st ISCIE Int. Symp. on Stochastic Systems Theory and Its Applications, pp. 87-94 (2009)

[12]S. Sugimoto, Y. Kubo and N. Munetomo: A quasilinear filter with conditional Gaussian sum distributions for nonlinear dynamical systems; Preprints of the 18th IFAC World Congress, pp. 7797-7802 (2011)

[13]G. H. Golub and J. H. Welsch: Calculation of Gauss quadrature rules; Math. Comput., Vol. 23, No. 106, pp. 221-230 (1969)

[14]G. H. Golub: Some modified matrix eigenvalue problems; SIAM Review, Vol. 15, No. 2, pp. 318-334 (1973)

[15]M. Arai: Note on Gauss quadrature (in Japanese); Private Comunication in Ritsumeikan Univ., Oct. (2012)

[16]D. L. Alspach and H. W. Sorenson: Nonlinear Bayesian estimation using Gaussian sum approximations; IEEE Trans. on Automatic Control, Vol. AC-17, No. 4, pp. 439-448 (1972)

[17]Y. Sunahara: An approximate method of state estimation for nonlinear dynamical systems; Trans. ASME, J. of Basic Engineering, Vol. 92D, No. 2, pp. 385-393 (1970)

[18]T. Katayama: Equivalent linearization Kalman filter with application to cubic sensor problems; Proc. of 12th European Control Conf., pp. 1633-1638 (2013)

[19]Y. C. Ho and R. C. K. Lee: A Bayesian approach to problems in stochastic estimation and control; IEEE Trans. on Automatic Control, Vol. AC-9, No. 4, pp. 333-339 (1964) 


\section{Authors}

\section{Sueo Sugimoto (Member)}

Sueo Sugimoto received the B.S. (1969)

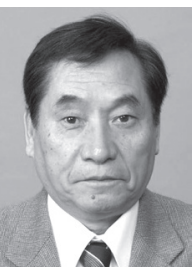

and M.S. (1971) degrees in mechanical engineering from the Kyoto Institute of Technology, Kyoto, Japan, and the Ph.D. (1974) degree in electrical engineering system science from the Polytechnic Institute of New York (presently, Polytechnic Institute of NYU), New York. He is a professor, Department of Electrical and Electronic Engineering at Ritsumeikan University, Shiga and Kyoto, Japan. His research interests include stochastic systems, and statistical image-signal processing in various applications such as GPS/GNSS navigation.

\section{Yukihiro KuBo (Member)}

Yukihiro KuBo received the B.S. (1997),

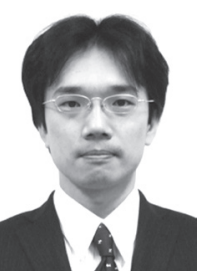
M.S. (1999) and Ph.D. (2002) degrees in Electrical and electronic engineering from Ritsumeikan University, Shiga and Kyoto, Japan. He worked in the production section of GPS car-navigation systems at Mitsubishi Electric Corp., Sanda Works from 2002 to 2004. He joined Department of Electrical and Electronic Engineering of Ritsumeikan University in 2004, and he is presently an associate professor. His research interests include GPS/GNSS signal processing and INS/GNSS integration systems.

Masaharu OHAshi (Student-Member)

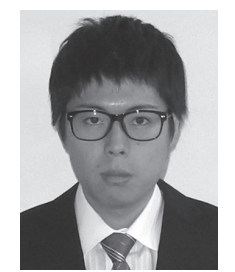

Masaharu OHASHI received the B.S. (2010) and M.S. (2012) degrees in Electrical and Electronic Engineering from Ritsumeikan University, Shiga, Japan in 2011. He is presently a Ph.D. student at graduate school ofScience and Engineering, Ritsumeikan University. His research interests include GPS/GNSS statistical signal processing. 